\title{
El dolor y su impacto en la calidad de vida y estado anímico de pacientes hospitalizados*
}

\author{
Pain and its Impact on the Quality of Life and \\ Mood of Hospitalized Patients
}

Enviado: diciembre 12 de 2011 | Revisado: junio 10 de 2012 | Aceptado: junio 30 de 2012

\author{
JuAn MÁXimo MOLINA LINDE ** \\ Universidad Pablo de Olavide, España \\ JANICE FigueROA RODRÍGUEZ*** \\ Pontificia Universidad Católica de Puerto Rico, \\ Puerto Rico \\ ANA FERnANdA URIBE RODRÍGUEZ **** \\ Universidad Pontificia Bolivariana, Colombia
}

\section{RES UMEN}

Mediante este estudio descriptivo transversal se pretendió conocer la rela-

Para citar este artículo: Molina, J. M., Figueroa, J. \& Uribe, A. F. (2013). El dolor y su impacto en la calidad de vida y estado anímico de pacientes hospitalizados. Universitas Psychologica, 12(1), 55-62.

* Artículo de investigación. Agradecimientos. Se agradece al personal administrativo del Hospital Virgen de las Nieves en Granada, España, por permitir que la investigación se llevara a cabo en sus instalaciones. Asimismo, se agradece a los pacientes que participaron en el estudio su colaboración en el proceso. Conflicto de intereses. No existen conflictos de intereses que puedan afectar el interés primordial y los objetivos de este artículo o la opinión profesional de los autores respecto al asunto. Contribuciones de los autores. Juan Máximo Molina Linde: desarrollo del concepto del estudio y plan; adquisición, análisis e interpretación de datos; elaboración de la metodología; revisión, corrección y aportes al manuscrito. Janice Figueroa Rodríguez: búsqueda, revisión e integración de literatura; desarrollo y discusión del tema; elaboración del manuscrito. Ana Fernanda Uribe: supervisión y revisión del manuscrito.

*** Correspondencia: Juan Máximo Molina Linde. Universidad Pablo de Olavide. Ctra. de Utrera, km. 1, Sevilla, 41013, España.E-mail: jmmollin@upo.es

**** Avda. Las Américas 2250, Ponce, Puerto Rico. Email: janice.figueroa@upb.edu.co

****** Autopista Piedecuesta, Kilómetro 7, Bucaramanga, Santander, Colombia. E-mail: anafernanda.uribe@ upb.edu.co ción entre la intensidad percibida del dolor, el estado de ánimo y la calidad de vida en pacientes hospitalizados. La muestra estuvo compuesta por 271 pacientes (133 hombres y 138 mujeres; edad $M=56.79$ ) de un hospital de tercer nivel en Granada, España. Se administró el Perfil de Salud de Nottingham (PSN), la Escala de Ansiedad y Depresión de Goldberg (EADG) y la Escala Verbal Simple (EVS). Los resultados reflejan un empeoramiento en cada una de las áreas de la calidad de vida evaluada con el PSN, así como en la ansiedad y depresión medidas con la EADG, conforme la intensidad del dolor aumenta. Finalmente, se discuten algunas recomendaciones basadas en los resultados.

Palabras clave autores

Dolor, estado de ánimo, ansiedad, depresión, calidad de vida.

Palabras clave descriptores

Cuestionarios, hospitalización, España.

\footnotetext{
A B S T R A C T

By means of this descriptive traverse study, it was sought to understand the existing relationship between the intensity of the perceived pain, the mood and the quality of life in hospitalized patients. The sample used in this study, consisted of 271 patients (133 men and 138 women; mean age 56.79) of a third level hospital in Granada, Spain. The Nottingham Health Profile (NHP), Goldberg's Anxiety and Depression Scale (GADS) and the Simple Verbal Scale were administered. As the intensity of the pain increases, the results reflected a worsening in each one of the areas of the quality of life when evaluated with the NHP; as well as in the anxiety and depression levels when measured using GADS. Finally, some recommendations are discussed based on the results obtained.

Key words authors

Pain, Mood, Anxiety, Depression, Quality of Life.

Key words plus

Questionnaires, Hospitalization, Spain.
} 


\section{Introducción}

Atender la calidad de vida de las personas implica considerar un sinnúmero de factores que permitan entender, de manera contextualizada, la situación particular que cada uno experimenta. En este trabajo se consideran algunos de esos aspectos, de manera conjunta, en pacientes hospitalizados: el estado anímico (niveles de ansiedad y depresión), el dolor (intensidad percibida) y la calidad de vida. En estudios como el de Alves-Apóstolo, Figueiredo, Cruz y Alves-Rodrigues (2011), se evidencia una asociación positiva y fuerte entre depresión, ansiedad y estrés. Marsiglia (2010), por su parte, plantea que "existe la convicción de que los factores psicológicos son importantes en la predisposición o el desencadenamiento, progresión o exacerbación de las enfermedades" (p. 6).

En esa misma línea, el estudio realizado por González, Landero y García (2009) reflejó una relación directa entre depresión, ansiedad y síntomas psicosomáticos en estudiantes universitarios del Norte de México. Otros estudios como el de Morales-Chávez, Robles, Jiménez y Morales-Romero (2007) evidencian la alta prevalencia de ansiedad y depresión en mujeres mexicanas con cáncer de mama. No obstante, se observa cómo diferentes estudios vinculan de una manera u otra el estado emocional afectivo y las enfermedades o padecimientos físicos. De manera tal que resulta fundamental atender el estado de ánimo de las personas, bien sea a modo de prevención de enfermedades físicas o a modo de apoyo y acompañamiento para aquellos pacientes que ya se encuentran hospitalizados.

García y Rodríguez (2007) señalan que existe un vínculo estrecho y recíproco entre la calidad de vida y la salud de las personas. Refiriéndose a la calidad de vida y las enfermedades crónicas, las autoras sostienen que:

La calidad de vida se ha convertido en un indicador de la evolución del estado de salud de estos pacientes, como expresión de una estrategia no sólo de prolongar la vida, sino también de aliviar los síntomas y mantener el funcionamiento vital. (p. 4)
Esto hace pertinente la necesidad de realizar investigaciones relacionadas con la calidad de vida de las personas, particularmente de pacientes hospitalizados. Diversos autores han trabajado el tema del dolor en sus escritos e investigaciones (González \& Moreno, 2007; López, Sánchez, Rodríguez \& Vázquez, 2007; Robaina, 2005; Vallejo, 2008; Villar, 2006). Villar (2006) sostiene que "aunque el dolor es algo muy subjetivo, entender las vías que subyacen para la percepción del dolor aporta muchas pistas para su mejor control" (p. 250). Según indica, el dolor no es experimentado por todas las personas de la misma manera, por lo que sugiere que se consideren los aspectos neurofisiológicos, hormonales, culturales, contextuales y psicológicos involucrados. Villar destaca, además, que "el dolor no puede [no debe] ser reducido a la neurofisiología o a la farmacogenética" (p. 253).

De acuerdo con González y Moreno (2007), "existe un elevado porcentaje de pacientes con un inadecuado manejo del dolor, lo que repercute en una mayor limitación en la realización de las actividades diarias" (p. 422). El dolor forma parte de la vida cotidiana de muchos seres humanos alrededor del mundo, muchos de los cuales se encuentran hospitalizados por diversas razones. Sin duda alguna, la calidad de vida de estos puede verse deteriorada por la intensidad del dolor que perciben o experimentan; asunto que debe ser atendido por el equipo multidisciplinario de las instituciones hospitalarias. La investigación que se presenta a continuación se realizó, precisamente, con la intención de aportar a la construcción de conocimientos en esta área y a la calidad de vida de las personas hospitalizadas.

Debido a lo anterior, el objetivo de esta investigacion fue conocer la relación entre la intensidad del dolor percibida, el estado de ánimo (ansiedad y depresión) y la calidad de vida en pacientes hospitalizados.

\section{Método}

Se realizó un estudio descriptivo de tipo transversal mediante metodología de encuesta, en el Hospital Universitario Virgen de las Nieves de Granada (España). La población de estudio se conformó 
con todos los pacientes ingresados al momento de la recolección de datos (pacientes pertenecientes a 812 camas) que cumplieran los siguientes criterios de inclusión: estar hospitalizado en el momento de la encuesta y presentar un estado de conciencia que permitiera la realización de la misma. Se excluyeron los pacientes ingresados en Unidad de Cuidados Intensivos (UCI), Reanimación, Psiquiatría y Unidad de Desintoxicación, por considerar que escapaban de los objetivos del trabajo. Asimismo, fueron excluidos aquellos diagnosticados con alguna enfermedad neurológica (tipo demencia) o psiquiátrica que hiciera dudar de la fiabilidad de sus respuestas.

\section{Recolección de datos}

Se llevó a cabo mediante cuestionario individualizado, dividido en tres partes; en la primera, se recopilaron los datos sociodemográficos (edad, sexo, nivel cultural, estado civil y situación familiar). En la segunda, se administró el Perfil de Salud de Nottingham (PSN) (Alonso, Antó \& Moreno, 1990) conjuntamente con la Escala Verbal Simple (EVS) para evaluar la intensidad del dolor experimentado durante la realización de la entrevista (ausente, leve, moderado, intenso e insoportable).

El PSN es un instrumento que se adecua mejor a la población de pacientes con una cierta afectación de su estado de salud, que a la población general. Por tanto, resulta un instrumento conveniente para el estudio. De hecho, en investigaciones recientes ha sido utilizado para abordar la calidad de vida en población geriátrica (Ordu et al., 2011). Está formado por 38 ítems que responden a seis dimensiones: energía, dolor, reacciones emocionales, sueño, aislamiento social y movilidad física.

\section{Administración}

El cuestionario puede aplicarse en formato autoadministrado, entrevista personal o telefónica y enviado por correo. En el estudio se realizó la entrevista personal, dadas las características de la población (elevada tasa de analfabetismo, edad avanzada). El tiempo promedio de administración de la prueba fue de aproximadamente 10 minutos.

\section{Puntuación en cada dimensión}

Para completar el cuestionario, el entrevistado debe contestar «sí», en caso de que el ítem preguntado refleje adecuadamente su estado actual o sentimiento, o «no», en cualquier otro caso. Las respuestas se codifican otorgando un 1 a la contestación de «sí» y un 0 a la contestación «no». Las respuestas afirmativas de cada dimensión se multiplican por el valor ponderal. Posteriormente, se suman y se transforman en una puntuación de 0 (mejor estado) a 100 (peor estado). Se identificó la puntuación total del cuestionario de cada paciente (variable denominada salud general). Pese a que este valor no ha sido validado, podría resultar de interés al momento de valorar globalmente a cada paciente. Ha sido utilizado para comparar el deterioro, además de en cada dimensión, según las distintas variables.

\section{Validez}

Los estudios comparativos llevados a cabo, tanto para población general como para pacientes, evidencian buenos resultados de validez tanto de constructo como de contenido (Alonso et al., 1990; McKenna, Hunt, McEwen \& Pope, 1984; Tabali, Jeschke, Dassen, Ostermann \& Heinze, 2011).

\section{Fiabilidad}

La consistencia interna del instrumento se cataloga como alta, aun cuando es menor de 0.9 para todas las dimensiones (Alonso, Prieto \& Antó, 1994; Badia, Alonso, Brosa \& Lock, 1994). En términos de test-retest también es alta para todas las dimensiones (Bowling, 1994). Por otra parte, la sensibilidad al cambio ha sido evaluada en diversos estudios observacionales, mostrando resultados adecuados (Alonso et al., 1990; McKenna et al., 1984).

Por último, en la tercera parte, se administró la Escala de Ansiedad y Depresión de Goldberg (EADG) (Goldberg, Bridges, Duncan-Jones \& Grayson, 1988), para detectar posibles signos y síntomas de estas psicopatologías. La EADG es tanto una prueba de detección, con usos asistenciales y epidemiológicos, como una guía del interrogatorio 
clínico. Es un test heteroadministrado que no solo orienta hacia el diagnóstico de ansiedad o depresión (o ambas en casos mixtos), con una sensibilidad del $83.1 \%$ y especificidad del $81.8 \%$, sino que discrimina entre ellos y dimensiona sus respectivas intensidades (Montón et al., 1993). Tanto la subescala de ansiedad como la de depresión constan de nueve preguntas. Sin embargo, las cinco últimas preguntas de cada subescala solo se formulan si hay respuestas afirmativas a las cuatro primeras preguntas, que son obligatorias. En las subescalas, se pide a la persona que reporte si durante las dos últimas semanas ha presentado alguno de los síntomas que se citan. En caso de que se obtengan dos o más puntos en la subescala de ansiedad, se culmina el interrogatorio con las nueve preguntas, mientras que en la subescala de depresión se continúa preguntando hasta el noveno ítem, ante tan solo una respuesta afirmativa. Los puntos de corte (cut-off) se sitúan en cuatro o más para el estudio de ansiedad y en dos o más en el de depresión, con puntuaciones más altas mientras más severo sea el problema (Pérez-Echevarría \& Zaragoza Working Group for the Study of Psychiatric and Psychosomatic Morbidity [GZEMPP], 1993). Aun cuando las preguntas son muy claras, las respuestas solo admiten la dicotomía, de manera que en ocasiones el paciente duda cuando se trata de coincidencias de leve intensidad. En tal caso, es el juicio del profesional el que valora la significación clínica de la respuesta. La utilización de este instrumento constituye una herramienta eficaz y sencilla para la labor que realizan los profesionales sanitarios (Magnavita, 2007).

\section{Cálculo del tamaño muestral}

Se calculó el número de pacientes necesarios para obtener una muestra significativa de la población hospitalizada. Se consideraron las siguientes premisas: una prevalencia de dolor en el $50 \%$ de los pacientes; una diferencia máxima de $10 \%$ entre la prevalencia del dolor en la muestra y en la población, y un nivel de significación estadística del $95 \%$. Se incluyó una muestra representativa de los pacientes hospitalizados en todas las unidades del Hospital Universitario Virgen de las Nieves. Si- guiendo la lista de camas disponibles en cada servicio, se seleccionaron los participantes mediante un muestreo aleatorio simple. Aquellos pacientes que por algún motivo de exclusión no entraron a formar parte del estudio fueron reemplazados por otros.

\section{Análisis estadístico}

La descripción de las variables se realizó mediante media, desviación estándar $(D E)$ y valores mínimo y máximo, para variables cuantitativas; para variables categóricas, se utilizaron frecuencias absolutas y relativas. La posible relación entre la variable independiente (intensidad del dolor durante la realización de la entrevista) y las variables dependientes (escalas PSN y EADG) se analizó mediante la prueba estadística ANOVA y el coeficiente $\eta^{2}$. Se propone un nivel de significación estadística para $p<0.05$. Por último, para el análisis estadístico se utilizó el programa SPSS versión 17.0 para Windows (SPSS Inc.).

\section{Resultados}

Se seleccionó una muestra compuesta por un total de 271 pacientes ingresados, con una edad media de 56.79 años (desviación estándar [DE], 18.22; recorrido de 16 a 89 años), y con una distribución por sexo similar de 133 hombres (49.1\%) y 138 mujeres (50.9\%). Con relación al estado civil, un $67.2 \%$ eran personas casadas; un $12.5 \%$, solteras; un $14.4 \%$, viudas; un $5.5 \%$, divorciadas o separadas y un $0.4 \%$ vivían en pareja. En cuanto al nivel cultural, un $14 \%$ de los participantes eran analfabetos; un $35.1 \%$, analfabetos funcionales; un $30.6 \%$, tenían estudios primarios; tan solo un $11.8 \%$, estudios secundarios y el $8.5 \%$, estudios superiores.

En la Tabla 1 se puede apreciar que existen diferencias significativas entre la intensidad del dolor medido con la EVS y el dolor evaluado con el PSN $(p<0.001)$, igualmente ocurre con el estado emocional $(p<0.001)$, las alteraciones en el sueño $(p<0.001)$, el aislamiento social $(p<0.001)$ y entre la intensidad del dolor y el estado de salud general $(p<0.001)$. Asimismo, se aprecian diferencias 


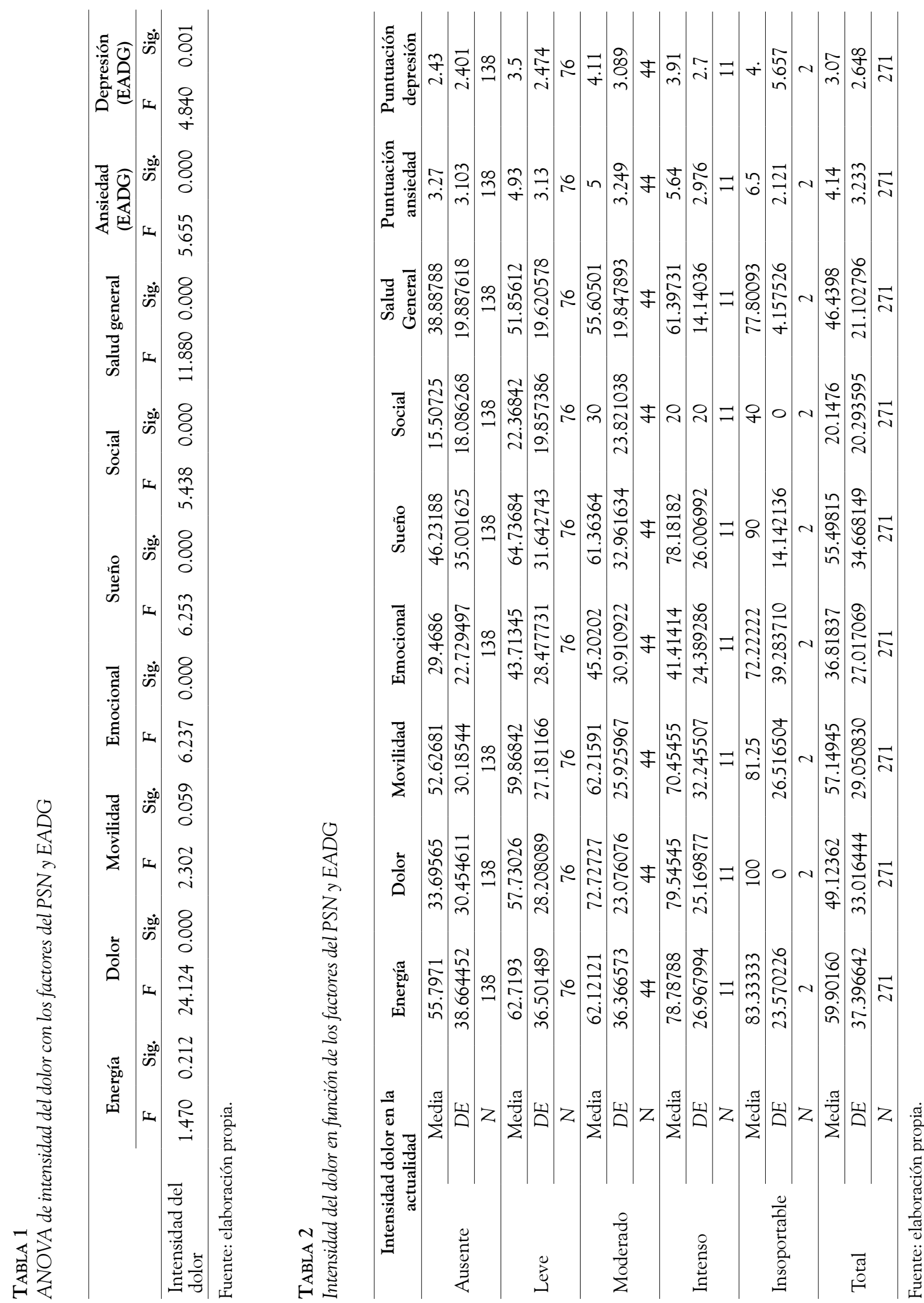


TABLA 3

Estadístico $\eta^{2}$ entre la intensidad del dolor con los factores del PSN y EADG

\begin{tabular}{lc}
\hline & $\mathbf{h}^{2}$ \\
\hline Energía * Intensidad dolor en la actualidad & 0.022 \\
Dolor * Intensidad dolor en la actualidad & 0.266 \\
Movilidad * Intensidad dolor en la actualidad & 0.033 \\
Emocional * Intensidad dolor en la actualidad & 0.086 \\
Sueño * Intensidad dolor en la actualidad & 0.086 \\
$\begin{array}{l}\text { Social * Intensidad dolor en la actualidad } \\
\text { Salud General * Intensidad dolor en la }\end{array}$ & 0.076 \\
$\begin{array}{l}\text { actualidad } \\
\text { Puntuación ansiedad * Intensidad dolor en la }\end{array}$ & 0.152 \\
$\begin{array}{l}\text { actualidad } \\
\text { Puntuación depresión * Intensidad dolor en }\end{array}$ & 0.078 \\
la actualidad & \\
\hline
\end{tabular}

Fuente: elaboración propia.

significativas entre la intensidad del dolor y los dos factores de la Escala de Goldberg; así, en ansiedad la significación es de $<0.001$ y en depresión, de 0.001 .

En la Tabla 2 se aprecia que, por lo general, existe un empeoramiento en cada una de las áreas de la calidad de vida evaluada con el PSN, así como en la ansiedad y depresión medidas con la escala EADG, conforme la intensidad del dolor aumenta.

Por otra parte, en la Tabla 3 se muestra, mediante el coeficiente $\eta^{2}$, el grado de asociación entre la intensidad del dolor y los distintos factores incluidos en el constructo de calidad de vida del PSN y la ansiedad y depresión de la escala EADG. Se aprecia que la intensidad del dolor influye mayormente en los factores dolor y salud general, medidos con el PSN.

\section{Discusión}

Se puede apreciar a través de este estudio cómo las distintas áreas evaluadas en las personas hospitalizadas, en lo que a ansiedad, depresión y calidad de vida se refiere, en general, fueron empeorando a medida que la intensidad del dolor percibido aumentaba. López et al. (2007) realizaron un estudio con pacientes consultantes de atención primaria. En el estudio se evidencia que "las personas que consultan por procesos dolorosos tienen su calidad de vida más deteriorada que las que consultan por otro tipo de procesos" (p. 18). Del mismo modo, se plantea que "el dolor crónico menoscaba la calidad de vida de una manera más amplia que el agudo" (p. 18). De manera tal que, en la búsqueda y promoción de la salud y el bienestar, se hace imprescindible trabajar con el dolor.

Vallejo (2008) señala la pertinencia de las técnicas de biofeedback, neurofeedback e hipnosis, en lo que al reconocimiento del dolor se refiere. De acuerdo con este autor, trabajar con los aspectos relacionados con el dolor de los pacientes, si bien no evitará el impacto del mismo en sus vidas, al menos podrá conseguir mejorar su situación. Según Vallejo, "recuperar el control de la propia vida traerá, por sí solo, una mejora en la autoestima personal y en la posibilidad de afrontar los problemas relacionados con la limitación impuesta con el dolor" (p. 426).

Considerando el impacto de las variables psicosociales en lo que respecta a la calidad de vida de personas que padecen enfermedades crónicas, Vinaccia y Orozco (2005) destacan que resulta fundamental atender las enfermedades crónicas desde un enfoque biopsicosocial, ambiental y cultural. En esa misma línea, Cano et al. (2006) señalan el vínculo existente entre las enfermedades crónicas, la situación socioeconómica y cultural de los pacientes y su impacto en la calidad de vida. Cardona y Agudelo (2005), por su parte, destacan la importancia de atender el concepto calidad de vida desde el plano individual y colectivo. Según plantean, lo individual involucra aspectos subjetivos importantes como la percepción. Sin embargo, señalan que atender la parte colectiva también resulta fundamental en tanto permite contextualizar la situación de las personas. Tener en cuenta el contexto permitirá una visión más clara y completa acerca de la realidad del paciente que, al mismo tiempo, facilitará el conocimiento de los recursos, herramientas, necesidades, fortalezas y limitaciones, para apoyarle de manera eficaz.

En este estudio se ve reflejada la asociación entre dos dimensiones del ser humano (física y emocional). Una alteración en cualquiera de estas áreas puede impactar la otra, afectando así la calidad de vida de las personas, en este caso, pacientes hospitalizados. Por tanto, como lo plantea Robaina (2005), 
las unidades de dolor deben ser multidisciplinarias. Un equipo de profesionales preparados para atender al ser humano en todas sus dimensiones podrá impactar mejor la calidad de vida de los pacientes hospitalizadas; lo cual a su vez influye en su proceso de recuperación, ofreciéndoles un acompañamiento que les permita asumir de manera saludable la situación que experimentan y construir estilos de vida que los apoyen en el proceso.

\section{Conclusiones}

Como parte de las gestiones que podrían realizarse para mejorar la calidad de vida de pacientes hospitalizados se deben incorporar terapias antiálgicas en los hospitales, de modo que al reducirse el malestar provocado por el dolor, la persona se encuentre en una posición que le permita enfrentarse a su situación en mejores condiciones y que, a su vez, facilite su proceso de recuperación.

En caso de que el dolor, por la naturaleza de la condición médica, no pueda ser eliminado es preciso realizar acompañamiento social y psicológico, de modo que estas dos últimas áreas se conviertan en una herramienta para manejar la situación.

El acompañamiento de familiares y seres queridos resulta fundamental en los procesos de recuperación tanto en el nivel físico como en el emocional. Por ende, los programas de psicoeducación a familiares y encargados son una alternativa que se debe considerar, si se pretende promover una mejor calidad de vida para las personas hospitalizadas.

Del mismo modo, en las instituciones hospitalarias se deben propiciar espacios de encuentro entre los pacientes, grupos de apoyo, orientaciones, actividades recreativas, así como visitas de familiares, grupos o asociaciones que les brinden apoyo social, emocional y espiritual. Todo esto tomando en cuenta la capacidad del paciente de involucrarse en tales actividades y las necesidades particulares de cada uno.

Las instituciones hospitalarias y los centros de salud deben contar con un equipo profesional multidisciplinario preparado para atender al ser humano en todas sus dimensiones, abordando tanto aspectos físicos como afectivo-emocionales, socioeconómicos, culturales, etc., y considerando las diferencias individuales.

\section{Referencias}

Alonso, J., Antó, J. M. \& Moreno, C. (1990). Spanish version of the Nottingham Health Profile: Translation and preliminary validity. American Journal of Public Health, 80(6), 704-708.

Alonso, J., Prieto, L. \& Antó, J. M. (1994). The Spanish version of the Nottingham Health Profile: A review of adaptation and instruments characteristics. Quality of Life Research, 3(6), 385-393.

Alves, J. L., Figueiredo, M. H., Cruz, A. \& Alves, M. (2011). Depression, anxiety and stress in primary health care users. Revista Latino-Americana de Enfermagem, 19(2), 348-353.

Badia, X., Alonso, J., Brosa, M. \& Lock, P. (1994). Reliability of the Spanish version of the Nottingham Health Profile in patients with stable end-stage renal disease. Social Science \& Medicine, 38(1), 153-158.

Baro, E., Ferrer, M., Vázquez, O., Miralles, R., Pont, A., Cervera, A., et al. (2006). Using the Nottingham Health Profile (NHP) among older adult inpatients with varying cognitive function. Quality of Life Research, 15(4), 575-585.

Bowling, A. (1994). La medida de la salud. Revisión de las escalas de medida de la calidad de vida. Barcelona: Editorial Masson.

Cano, E., Quiceno, J., Vinaccia, S., Gaviria, A. M., Tobón, S. \& Sandín, B. (2006). Calidad de vida y factores psicológicos asociados en pacientes con diagnóstico de dispepsia funcional. Universitas Psychologica, 5(3), 511-520.

Cardona, D. \& Agudelo, H. B. (2005). Construcción cultural del concepto calidad de vida. Revista Facultad Nacional de Salud Pública, 23(1), 79-90.

García, C. R. V. \& Rodríguez, G. (2007). Calidad de vida en enfermos crónicos. Revista Habanera de Ciencias Médicas, 6(4), 1-9.

Goldberg, D., Bridges, K., Duncan-Jones, P. \& Grayson, D. (1988). Detecting anxiety and depression in general medical settings. British Medical Journal, 297(6653), 897-899. 
González, C. \& Moreno, M. G. (2007). Manejo del dolor crónico y limitación en las actividades de la vida diaria. Revista de la Sociedad Española del Dolor, 14(6), 422-427.

González, M. T., Landero, R. \& García, J. (2009). Relación entre la depresión, la ansiedad y los síntomas psicosomáticos en una muestra de estudiantes universitarios del norte de México. Revista Panamericana de Salud Pública/Pan American Journal of Public Health, 25(2), 141-145.

López, M. C., Sánchez, M., Rodríguez, M. C. \& Vázquez, E. (2007). Cavidol: calidad de vida y dolor en atención primaria. Revista de la Sociedad Española del Dolor, 14(1), 9-19.

McKenna, S. P., Hunt, S. M., McEwen, J. \& Pope C. (1984). Changes in the perceived health of patients recovering from fractures. Public Health, 98(2), 97-102.

Marsiglia, I. (2010). Estudio clínico y epidemiológico de la ansiedad y la depresión en una muestra de 1.159 pacientes. Gaceta Médica de Caracas, 118(1), 3-10.

Montón, C., Pérez-Echevarría, M. J., Campos, R., García-Campayo, J., Lobo, A. \& Zaragoza Working Group for the Study of Psychiatric and Psychosomatic Morbidity (1993). Escalas de ansiedad y depresión de Goldberg: una guía de entrevista eficaz para la detección del malestar psíquico. Atención Primaria, 12(6), 345-349.

Magnavita, N. (2007). Anxiety and depression at work. The A/D Goldberg Questionnaire. Giornale Italiano di Medicina del Lavoro ed Ergonomia, 29(3), 670-671.

Morales, M., Robles, R., Jiménez, M. \& Morales, J. (2007). Las mujeres mexicanas con cáncer de mama presentan una alta prevalencia de depresión y ansiedad. Salud Pública de México, 49(4), 247-248.

Ordu, N. K., Gokce-Kutsal, Y., Borman, P., Ceceli, E., Dogan, A., Eyigor, S., et al. (2011). Pain and quality of life (QoL) in elderly: The Turkish experience. Archives of Gerontology and Geriatrics, 55(2), 357 362. doi:10.1016/j.archger.2011.10.019

Pérez-Echevarría, M. J. \& Zaragoza Working Group for the Study of Psychiatric and Psychosomatic Morbidity (1993, noviembre). Evaluación multiaxial de pacientes en Atención Primaria: el estudio epidemiológico de Zaragoza. XXIX Congreso Nacional de la Sociedad Española de Medicina Psicosomática, Zaragoza, España.

Robaina, F. J. (2005). ¿Por qué las unidades del dolor deben sermultidisciplinarias? Revista de la Sociedad Española del Dolor, 12(3), 137-140.

Tabali, M., Jeschke, E., Dassen, T., Ostermann, T. \& Heinze, C. (2011). The Nottingham Health Profile: A feasible questionnaire for nursing home residents? International Psychogeriatrics, 24(3), 416-424. doi: $10.1017 / S 1041610211001931$

Vallejo, M. A. (2008). Perspectivas en el estudio y tratamiento psicológico del dolor crónico. Clínica y Salud, 19(3), 417- 430.

Villar, J. (2006). Cómo investigar en algo tan subjetivo como el dolor. Revista de la Sociedad Española del Dolor, 13(4), 250-253.

Vinaccia, S. \& Orozco, L. M. (2005). Aspectos psicosociales asociados con la calidad de vida de personas con enfermedades crónicas. Diversitas: Perspectivas en Psicología, 1(2), 125-137. 